\title{
A NOTE ON CHAIN CONDITIONS IN NILPOTENT RINGS AND GROUPS
}

\author{
S. A. JENNINGS
}

Maximal and minimal conditions for ideals in associative rings have of ten been considered, but little seems to be known of these conditions in non-associative rings, or of chain conditions on the non-normal subgroups of a group. Moreover, it is usual to assume the condition for one-sided ideals in noncommutative rings, and the weaker condition for two-sided ideals rarely appears. In this note we first consider a class of groups which are "nilpotent" with respect to a set of operators $\Omega$. These groups include ordinary nilpotent groups, and associative and non-associative nilpotent rings and algebras as special cases. Our main theorem is to the effect that, for an $\Omega$-nilpotent group, the maximal or minimal condition for $\Omega$-subgroups implies the corresponding condition for all subgroups. As immediate consequences of this theorem it follows that, for nilpotent rings and algebras, the maximal or minimal condition for ideals implies the corresponding condition for modules, while for nilpotent groups, the maximal or minimal condition for normal subgroups implies the corresponding condition for all subgroups.

1. Definition of $\Omega$-nilpotency. Let $R$ be a group, and let $\Omega$ be a set of (left) operators of $R$ which includes the inner automorphisms of $R$. $R$ may also admit a second set of operators $\Phi$, in which case it will be understood in what follows that all subgroups are supposed to admit $\Phi$. The statement that a certain subgroup is an $\Omega$-subgroup means that this subgroup admits $\Omega$ as well as $\Phi$.

If $A$ is any $\Omega$-subgroup of $R, A$ is normal, and we may suppose that $R / A$ admits the same sets of operators, $\Omega$ and $\Phi$, as $R$.

We take the following as our

DEFINITION OF AN $\Omega$-NILPOTENT GROUP. ${ }^{1} R$ is $\Omega$-nilpotent if there exists a strictly decreasing chain of $\Omega$-subgroups

Presented to the Royal Society of Canada, May 25, 1943, and to the American Mathematical Society, April 29, 1944; received by the editors January 21, 1944, and, in revised form, March 18, 1944.

1 Originally I discussed the chain theorems for groups for and rings separately, and while it was clear that the results were analogous, I did not succeed in unifying the proofs for the two cases. The referee, Dr. Irving Kaplansky, pointed out, however, that this could be accomplished by the use of the general notion of $\Omega$-nilpotency which we give here, and which was suggested by him. I would like to express my thanks to the referee for his contribution to this paper. 


$$
R=A_{1} \supset A_{2} \supset A_{3} \supset \cdots \supset A_{m} \supset A_{m+1}=1,
$$

terminating with the identity, and such that if $\lambda \in \Omega$ and $a_{i} \in A_{i}$ then

$$
a_{i}^{-r}\left(\lambda a_{i}\right) \in A_{i+1}, \quad i=1,2, \cdots, m,
$$

for some $r$, where in general $r$ may be any positive or negative integer, or zero, but if $\lambda$ happens to be an inner automorphism then $r$ must equal one.

The minimum length of all chains satisfying (1) and (2) above will be called the exponent of $R$ and any chain of $\Omega$-subgroups satisfying these conditions and having minimum length will be called a minimum chain.

The following results are needed for our later induction.

LEMMA A. If $R$ is an $\Omega$-nilpotent group of exponent $m$, and

$$
R=A_{1} \supset A_{2} \supset \cdots \supset A_{m} \supset A_{m+1}=1
$$

is a minimum chain, then $R / A_{m}$ is an $\Omega$-nilpotent group of exponent $m-1$.

Lemma B. The subgroup $A_{m}$ of any chain satisfying (1) and (2) above is contained in the centre of $R$. Moreover, every subgroup of $A_{m}$ is an $\Omega$-subgroup of $R$.

Lemma C. If $A$ is any $\Omega$-subgroup of $R$, then the maximal or minimal condition for $\Omega$-subgroups of $R$ implies the corresponding condition for $\Omega$-subgroups in $R / A$.

Lemmas $\mathrm{A}$ and $\mathrm{C}$ may be readily verified. To prove Lemma $\mathrm{B}$, we note that if $a_{m} \in A_{m}, \lambda \in \Omega$, then $a_{m}^{-r}\left(\lambda a_{m}\right)=1$, since $A_{m+1}=1$, and hence $\lambda a_{m}=a_{m}^{r}$, which implies that every subgroup of $A_{m}$ admits $\Omega$. Again, if $x \in R, x^{-1} a_{m} x=a_{m}$, since for inner automorphisms $r=1$, and hence very element $a_{m}$ of $A_{m}$ is in the centre of $R$.

2. The chain theorem. We are now in a position to prove the following

THEOREM. Let $R$ be an $\Omega$-nilpotent group. The maximal or minimal condition for $\Omega$-subgroups in $R$ implies the maximal or minimal condition respectively for all subgroups in $R$.

We prove that if the maximal conditions for $\Omega$-subgroups holds in $R$, the maximal condition for all subgroups also holds; the corresponding result for the minimal condition may be proved in a similar way.

Suppose that $R$ has exponent $m$, and satisfies the maximal condi- 
tion for $\Omega$-subgroups. If $m=1$, our result is true by Lemma $\mathrm{B}$. We proceed by induction and assume that the result holds for groups with exponent at most $m-1$, and hence, by Lemma $C$ and Lemma $A$, for $\bar{R}=R / A_{m}$. Let

$$
M_{1} \subseteq M_{2} \subseteq M_{3} \subseteq \cdots
$$

be an ascending chain of subgroups of $R$, and let $\bar{M}_{i}=\left(M_{i} \cup A_{m}\right) / A_{m}$ be the image in $\bar{R}$ of $M_{i}$. We have clearly

$$
\bar{M}_{1} \subseteq \bar{M}_{2} \subseteq \bar{M}_{3} \subseteq \cdots \text {, }
$$

an ascending chain of subgroups in $\bar{R}$. Now the maximal condition for subgroups holds in $\bar{R}$, since the condition for $\Omega$-subgroups holds in $\bar{R}$ by Lemma $C$, and hence there exists an integer $j$ such that

$$
\bar{M}_{i}=\bar{M}_{i+1}
$$

for $i \geqq j$.

This implies that

$$
M_{i} \cup A_{m}=M_{i+1} \cup A_{m} \quad \text { for } i \geqq j .
$$

Consider $N_{i}=M_{i} \cap A_{m}$; clearly $N_{i}$ is a subgroup of $A_{m}$, and hence by Lemma $\mathrm{B}$ is an $\Omega$-subgroup of $R$, and we have

$$
N_{1} \subseteq N_{2} \subseteq N_{3} \subseteq \cdots,
$$

an ascending chain of $\Omega$-subgroups of $R$. Hence there exists an integer $k$ such that

$$
N_{i}=N_{i+1} \quad \text { for } i \geqq k .
$$

Under our hypothesis, therefore, we see that, provided $i \geqq \max (j, k)$,

$$
\begin{aligned}
M_{i} & \subseteq M_{i+1}, \\
M_{i} \cup A_{m} & =M_{i+1} \cup A_{m}, \\
M_{i} \cap A_{m} & =M_{i+1} \cap A_{m} .
\end{aligned}
$$

Now $A_{m}$ belongs to the centre of $R$, and hence $M_{i}$ is normal in $M_{i} \cup A_{m}$, and therefore normal in $M_{i+1} \cup A_{m}$ : also $M_{i+1}$ is normal in $M_{i+1} \cup A_{m}$. Therefore the five subgroups $M_{i}, M_{i+1}, M_{i} \cup A_{m}$ $=M_{i+1} \cup A_{m}, M_{i} \cap A_{m}=M_{i+1} \cap A_{m}, A_{m}$ are all normal subgroups of $M_{i+1} \cup A_{m}$, and hence form a modular lattice, and hence the conditions (3) above imply $M_{i}=M_{i+1}$, which proves our theorem.

3. The chain theorem of nilpotent groups. Consider now the case where $R$ is a nilpotent group in the usual sense. ${ }^{2}$ It is easily seen that, if $\Omega$ is the set of inner automorphisms, $R$ is $\Omega$-nilpotent. For let

\footnotetext{
2 For definitions and properties of nilpotent groups see, for example, H. Zassenhaus, Gruppentheorie, vol. 1, Leipzig, 1937, pp. 118-119.
} 


$$
R=A_{1} \supset A_{2} \supset \cdots \supset A_{m} \supset A_{m+1}=1
$$

be any strictly decreasing central series of $R$ (for example, the lower central series); we have always, if $a_{i} \in A_{i}, x \in R$,

$$
\left(a_{i}^{-1} x^{-1} a_{i} x\right) \in A_{i+1}
$$

which is precisely condition (2) above applied to an inner automorphism, with $r=1$. Since the $\Omega$-subgroups are precisely the normal subgroups of $R$, we have:

"In a nilpotent group the maximal or minimal condition for normal subgroups implies the corresponding condition for all subgroups."

4. The chain theorem for nilpotent rings. The definition of nilpotency is not well established for general non-associative rings. We give here a definition which applies to any ring, associative or not, and which is equivalent to the familiar one in the case of associative and Lie rings. This particular definition seems natural, and the definition of an $\Omega$-nilpotent group as given earlier is a further generalization.

We shall say that $R$ is a generalized ring, or briefly a ring, which admits $\Phi$ as a set of (left) operators if the following conditions hold:

(a) $R$ is an abelian group which admits $\Phi$.

(b) If $a, x \in R$, then $a x \in R$, that is, $R$ is closed under multiplication.

(c) If $a, b, x \in R$, then $x(a+b)=x a+x b,(a+b) x=a x+b x$, that is, the multiplications are left and right operators of $R$.

It is clear that associative and Lie rings and algebras, and hypercomplex systems both associative and non-associative, are rings in the above sense. We may also suppose that $\Phi$ is not empty, since the rational integers may always be adjoined to any domain of operators. By a module of $R$ we mean an additive subgroup of $R$ which admits $\Phi$, that is, a module is a $\Phi$-subgroup. By a left, right or two-sided ideal of $R$ we mean a module which admits the elements of $R$ as left, right or two-sided operators respectively. Unless otherwise stated, we shall understand by ideal a two-sided ideal.

If $A$ is any ideal of $R$, the residue classes modulo $A$ form a generalized ring $R / A$ which is homomorphic to $R$, and we may suppose that $R$ and $R / A$ have the same set of operators.

Because our rings are not necessarily associative, we take the following as our

Definition of A NILPOTENT RING. $A$ generalized ring $R$ will be said to be nilpotent if it has a strictly decreasing chain of ideals

$$
R=A_{1} \supset A_{2} \supset A_{3} \supset \cdots \supset A_{m} \supset A_{m+1}=0,
$$


terminating with zero, such that if $x \in R$ and $a_{i} \in A_{i}$ then

$$
x a_{i} \in A_{i+1}, \quad a_{i} x \in A_{i+1} .
$$

If $R$ is an associative or Lie ring or algebra, the above definition of nilpotency is obviously equivalent to the usual one.

It is clear that a generalized ring which is nilpotent as defined above is an $\Omega$-nilpotent abelian group, where $\Omega$ consists of the right and left multiplications of $R$, and the integer $r$ of condition (2) above always equals zero. The $\Omega$-subgroups are precisely the ideals of $R$, and our earlier theorem implies that

"In any generalized nilpotent ring (and in particular, in any nilpotent associative or Lie ring or algebra) the maximal or minimal condition for ideals implies the corresponding condition for modules."

The University of British Columbia 